\title{
Large magnetocaloric effects in magnetic intermetallics: First-principles and Monte Carlo studies
}

\author{
Peter Entel ${ }^{1, a}$, Markus E. Gruner ${ }^{1,2}$, Masako Ogura ${ }^{3}$, Vladimir V. Sokolovskiy ${ }^{4}$, Vasiliy D. Buchelnikov ${ }^{4}$, Anna Grünebohm $^{1}$, \\ Raymundo Arróyave ${ }^{5}$, Kazuhiko Uebayashi ${ }^{6}$, Navdeep Singh ${ }^{7}$, Anjana Talapatra ${ }^{5}$, Thien Duong ${ }^{5}$, Mehmet Acet ${ }^{1}$ and Aslı \\ Çakır ${ }^{1,8}$ \\ ${ }^{1}$ University of Duisburg-Essen, Faculty of Physics and CENIDE, 47048 Duisburg, Germany \\ ${ }_{2}^{2}$ Forschungs-Neutronenquelle Heinz-Maier-Leibnitz (FRM II), TU München, 65748 Garching, Germany \\ ${ }^{3}$ Department of Physics, Osaka University, Machikaneyama 1-1, Osaka 560-0043, Japan \\ ${ }^{4}$ Condensed Matter Physics Department, Chelyabinsk State University, 454021 Chelyabinsk, Russia \\ ${ }^{5}$ Department of Materials Science and Engineering, University of Texas A\&M, College Station, Texas 77843, USA \\ ${ }^{6}$ NIT, Akita College, Akita, Japan \\ ${ }^{7}$ Department of Engineering and Technology, University of Houston, Houston, Texas 77204, USA \\ ${ }^{8}$ Metallurgical and Materials Engineering Department, Mugla University, Mugla, Turkey
}

\begin{abstract}
We have performed ab initio electronic structure calculations and Monte Carlo simulations of frustrated ferroic materials where complex magnetic configurations and chemical disorder lead to rich phase diagrams. With lowering of temperature, we find a ferromagnetic phase which transforms to an antiferromagnetic phase at the magnetostructural (martensitic) phase transition and to a cluster spin glass at still lower temperatures. The Heusler alloys $\mathrm{Ni}-(\mathrm{Co})-\mathrm{Mn}-(\mathrm{Cr})-(\mathrm{Ga}, \mathrm{Al}, \mathrm{In}, \mathrm{Sn}, \mathrm{Sb})$ are of particular interest because of their large inverse magnetocaloric effect associated with the magnetostructural transition and the influence of $\mathrm{Co} / \mathrm{Cr}$ doping. Besides spin glass features, strain glass behavior has been observed in Ni-Co-Mn-In. The numerical simulations allow a complete characterization of the frustrated ferroic materials including the Fe-Rh-Pd alloys.
\end{abstract}

\section{Introduction}

The widely used intermetallics Ti-Ni have attracted much interest over decades because of their martensitic transformation, superelasticity and excellent shape memory behavior [1] and new glass phases [2]. With magnetism as additional degree of freedom, magnetic shape memory Heusler alloys have been found with large reversible deformations under the application or removal of a magnetic field [3]. The strong coupling between magnetism and structure leads to a first order magnetostructural transition with different magnetization of austenite and martensite because of different magnetocrystalline anisotropy and different magnetic exchange coupling constants. The stronger anisotropy of the martensitic phase allows rotation of the martensitic variants under the application of a magnetic field, leading to the magnetic shape memory effect [4]. Magnetic superelasticity is involved in the stabilization of the phase with highest magnetization in an external magnetic field $[5,6]$. In addition to shape memory and superelasticity, these alloys (ternary magnetic shape memory Heusler alloys of type $\mathrm{Ni}-(\mathrm{Co})-\mathrm{Mn}-(\mathrm{Cr})-(\mathrm{Al}, \mathrm{Ga}, \mathrm{In}, \mathrm{Sn}, \mathrm{Sb})$ ) exhibit magnetocaloric (conventional and inverse) [7, 8, 9], barocaloric [10] and elastocaloric [11] effects, magnetoresistance [12], exchange bias [13] and kinetic arrest [14]. Also spin-glass [15] and strain-glass [16]

a Corresponding author: entel@thp.uni-duisburg.de features have been reported on several of these Heusler alloys.

In this short communication, we compare the structural and magnetic behavior and magnetocaloric properties of cubic Heusler alloys shown in Fig. 1 with some of the giant magnetocaloric materials known before like $\mathrm{Gd}_{5}\left(\mathrm{Si}_{2} \mathrm{Ge}\right)_{2}$ (monoclinic crystal structure) [17], $\mathrm{La}\left(\mathrm{Fe}_{13-\mathrm{x}} \mathrm{Si}_{\mathrm{x}}\right)$ (cubic $\mathrm{NaZn}_{13} \mathrm{D}_{3}$-type structure) [18], and Fe-Rh (cubic CsCl-type structure) [19]. The comparison shows remarkable similarities associated with the inverse magnetocaloric effect emerging from the magnetostructural transition in the magnetic Heusler alloys with the corresponding magnetostructural transition and caloric effect of the Gd based and Fe-Rh-Pd based compounds as discussed below.

\section{Structural and magnetic phase diagrams of Heusler intermetallics}

Figure 1 shows the intersection of structural and magnetic phase transitions in the phase diagrams of Ni-Mn-Ga and $\mathrm{Ni}-\mathrm{Mn}-\mathrm{In}$ as a function of the valence electron concentration $[20,21]$. The $a b$ initio calculations in combination with Monte Carlo simulations reproduce fairly well the experimental phase diagrams, which have been discussed in [3]. 


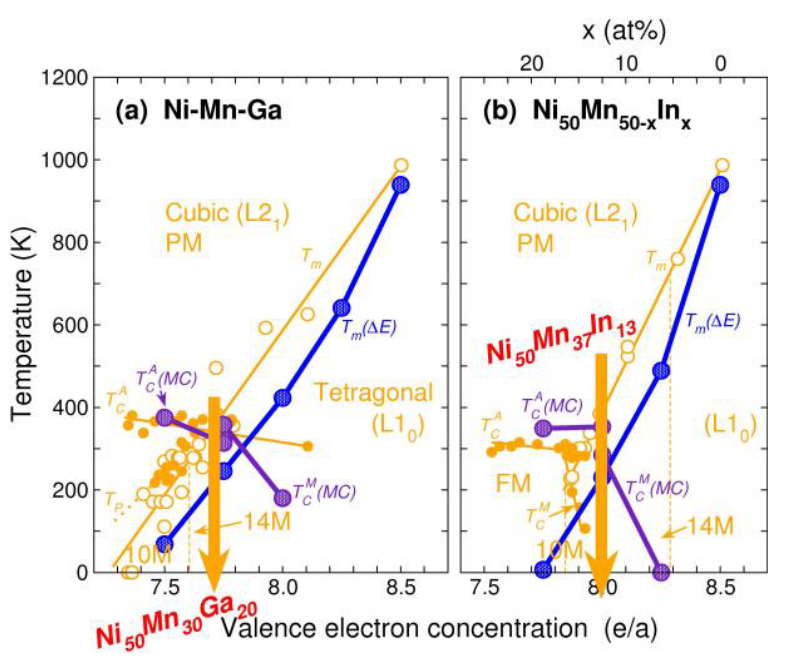

Figure 1. Phase diagrams of Ni-Mn-Ga and Ni-Mn-In in the temperature versus electron concentration plane in comparison with experimental data (open circles mark the martensitic transformation temperature $M_{S}$ ) taken from various sources, see [3]. Dark lines (blue, indigo) mark martensitic $\mathrm{L}_{2}-\mathrm{L} 1_{0}$ transformation temperatures (from $a b$ initio total energy differences between austenite and martensite [20, 21]) and Curie temperatures (from Monte Carlo simulations using $a b$ initio magnetic exchange coupling constants [20, 21]), respectively. Recent a $b$ initio calculations have been done along the path marked by the arrow in each phase diagram.

Special attention is given in this paper to the magnetic properties of the non-stoichiometric Ni-Mn-Ga and NiMn-In Heusler alloys (see [22] for the case of $\left.\mathrm{Ni}_{50} \mathrm{Mn}_{34} \mathrm{In}_{16}\right)$.

Figure 2 shows that the materials do not form homogeneous alloys, instead we observe a multiplicity of coexisting non-modulated $\left(\mathrm{L}_{0}\right)$ and modulated $(5 \mathrm{M}, 7 \mathrm{M})$ intermediate martensitic structures because of chemical clustering effects in the alloys [23, 24]. This also leads to multiple spin configurations. Especially in the case of $\mathrm{Mn}$ - excess in $\mathrm{X}_{2} \mathrm{Y}_{1+\mathrm{x}} \mathrm{Z}_{1-\mathrm{x}}$ alloys, where the excess $\mathrm{Mn}_{\mathrm{Y}}$ atoms mostly go onto the $\mathrm{Z}$ sites, which is accompanied by a spin flip transition because of the antiferromagnetic exchange coupling constant between $\mathrm{Mn}_{\mathrm{Y}}$ on its original sublattice and $\mathrm{Mn}_{\mathrm{Z}}$ on the $\mathrm{Z}$ sublattice. This is because the distance between nearest neighbor $\mathrm{Mn}$ atoms on the Mn sublattice is $a \sqrt{ } 2 / 2$, where $a$ is the lattice constant of the cubic L2 $2_{1}$ structure, causing ferromagnetic order, which is reduced to $a / 2$ for nearest neighbor $\mathrm{Mn}_{\mathrm{Y}}$ and $\mathrm{Mn}_{\mathrm{Z}}$ atoms, causing antiferromagnetic order. Since the magnetic moment of $\mathrm{Mn}$ is $\approx 4 \mu_{\mathrm{B}}$, this spin flip has a large effect and leads to metamagnetic behavior of the Mn-based Heusler alloys (see also [21, 25]). This is discussed in more detail below.

\section{Energy variation of magnetic Heusler alloys along the Bain path}

Figure 3 shows the Energy variation, E(c/a), at zero temperature of $\mathrm{Ni}_{50} \mathrm{Mn}_{31.25} \mathrm{Ga}_{218.75}$ (using a $\mathrm{Ni}_{8} \mathrm{Mn}_{5} \mathrm{Ga}_{3}$ supercell to approximate the alloy) and the effect of $\mathrm{Co}$ doping using a $\mathrm{Ni}_{7} \mathrm{Co}_{1} \mathrm{Mn}_{5} \mathrm{Ga}_{3}$ supercell [24] along the Bain path showing the stability of the $\mathrm{Ll}_{0}$ martensitic phase at low temperature.

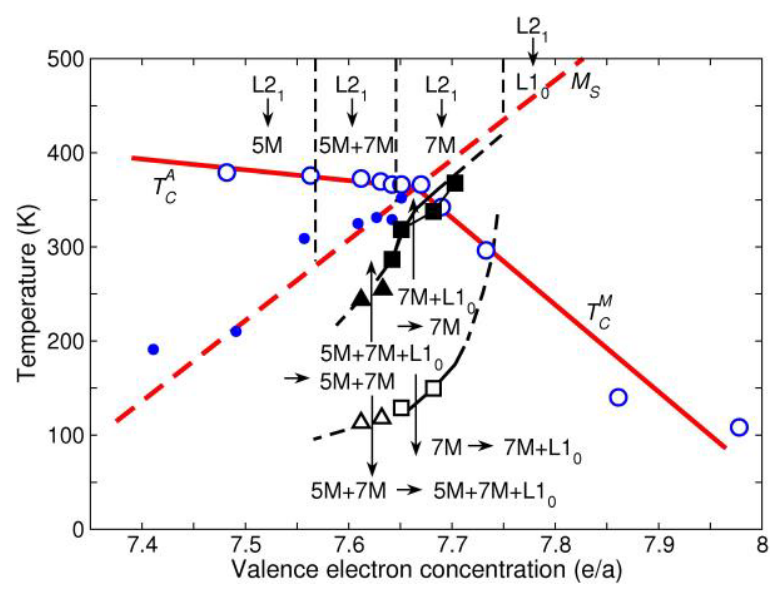

Figure 2. More detailed magnetic and structural phase diagram of Ni-Mn-Ga based on an alloy series with 50 at.\% fixed $\mathrm{Ni}$ concentration. The forward and reverse martensitic transformation lines are shown with the down and up-pointing arrows, respectively. Filled circles: $M_{S}$, open circles mark the Curie temperatures of autenite and martensite. Plotted from the data in [23].

With increasing temperature (which can be simulated using the fixed spin moment method allowing to calculate binding curves at reduced magnetization) "ferro" and "ferri" solutions move relative to each other until the "ferro" solution becomes lower in energy at the martensitic to austenitic transition.

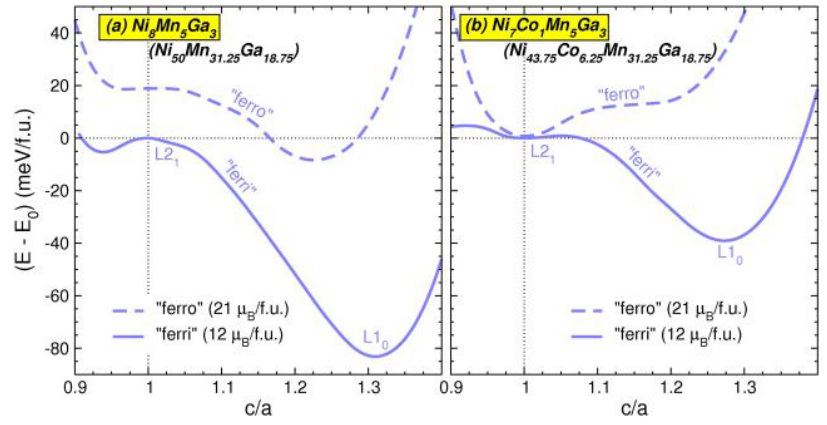

Figure 3. Total energy variation of $\mathrm{Ni}_{8} \mathrm{Mn}_{5} \mathrm{Ga}_{3}$ and $\mathrm{Ni}_{7} \mathrm{Co}_{1} \mathrm{Mn}_{5} \mathrm{Ga}_{3}$ (16-atom supercell) as a function of tetragonal distortion $c / a$ where $c / a=1$ is cubic austenite and $c / a>1$ is tetragonal martensite. Energies in (a) and (b) are relative to the "ferri" solution corresponding to the spin reversal of Mn spins on the Ga lattice sites which is lowest in energy in (a) and (b). "ferro" denotes the ferromagnetic solution with all spins parallel. Doping with Co weakens the stability of the martensitic structure as the minimum of $\mathrm{L}_{0}$ in (b) moves up in energy compared to (a). See also [24].

The magnetic exchange coupling constants can be obtained from ab initio calculations [24] and are plotted in Fig. 4 for the case of Mn-excess $\mathrm{Ni}_{50} \mathrm{Mn}_{30} \mathrm{Ga}_{20}$ compared to stoichiometric $\mathrm{Ni}_{2} \mathrm{MnGa}$. The chemical disorder caused by $\mathrm{Mn}$ excess introduces negative magnetic coupling constants (antiferromagnetic exchange interactions) which weaken the ferromagnetic spin configuration and lead to magnetic clustering effects which have been detected by neutron scattering experiments [25]. $\mathrm{Ni}_{50-\mathrm{x}} \mathrm{Co}_{\mathrm{x}} \mathrm{Mn}_{40} \mathrm{Sn}_{10}$ reveals an unusually large change (jump) of $M(T)$ at the martensitic transition: 
Ferromagnetism in the austenitic phase and "ferri" (spins on the $\mathrm{Mn}_{\mathrm{Z}}$ sublattice are reversed), antiferromagnetism or parmagnetism in the martensitic phase [26, 27]. There are ferromagnetic spin clusters of nanoscopic dimensions with center-to-center spacing around $12 \mathrm{~nm}$ [25]. This is the origin of metamagnetic behavior since a magnetic field applied to martensite can yield a first order transition to the ferromagnetic austenitic phase (see, for example, [28]). When adding Co to the Heusler alloys for getting better magnetocaloric effects, the magnetostructural transition is shifted to lower temperatures because of decrease of $e / a$ and eventually the martensitic phase vanishes altogether for larger Co concentration.
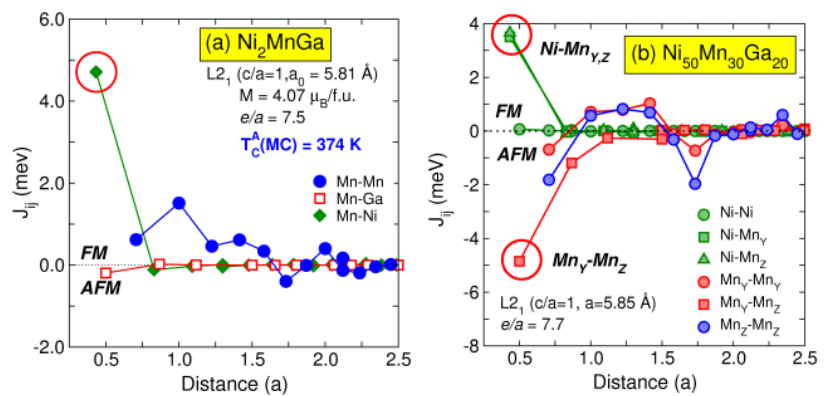

Figure 4. Element resolved magnetic coupling constants of (a) stoichiometric $\mathrm{Ni}_{2} \mathrm{MnGa}$ and (b) Mn-rich $\mathrm{Ni}_{50} \mathrm{Mn}_{30} \mathrm{Ga}_{20}$. In each case, the first atom of the labelled pair of atoms is sitting at the origin and the variation of the coupling constants is shown as a function of the distance between the atoms in the cubic $\mathrm{L} 2{ }_{1}$ structure. In martensite, the AFM contributions start to dominate (not shown here). Please, note that the Ni-Mn $\mathrm{M}_{\mathrm{Z}}$ and $\mathrm{Mn}_{\mathrm{Y}}-\mathrm{Mn}_{\mathrm{Z}}$ exchange interactions nearly cancel each other which may be considered as the microscopic origin of metamagnetism since it facilitates the large change of $M(T)$ at the magnetostructural transition [29]. Ni-In and Ni-Sn based Heusler alloys show similar behavior.

\section{Metamagnetic transition in Fe-Rh and $\mathrm{Ni}-\mathrm{Mn}-(\mathrm{Al}, \mathrm{Ga}, \mathrm{In}, \mathrm{Sn}, \mathrm{Sb})$ intermetallics}

We have recently argued that the phenomenon of metamagnetism in Heusler alloys can be understood by considering the complex spin configurations which appear due to spin-spin interactions arising from the orbital-resolved magnetic exchange coupling constants [29]. We have observed that in austenite, the ferromagnetic exchange interactions (between the $t_{2 g}$ orbitals of the $\mathrm{Mn}$ atoms on their original sublattice sites) can nearly be compensated by the antiferromagnetic exchange interactions associated with the $e_{g}$ orbitals [29, 30]. This feature appears in all magnetic Heusler alloys of the NiMn based series [30] (see the highlighted $J_{i j}$ values in Fig. 4(b) and also in Fig. 1 in [29]). When the alloy undergoes a martensitic transformation, the tetragonal distortion changes the distances between the atoms leading to an antiferromagnetic ground state with very low magnetization and spin-glass behavior at low temperatures for the Mn-rich alloys (because of chemical disorder and competing ferro- and antiferromagnetic exchange interactions in the alloy). The large change of magnetization is shown in Fig. 5 for the case of Ni-CoMn-Ga-In [26, 27]. This extraordinary behavior is displayed by other Heusler alloys, too [29].

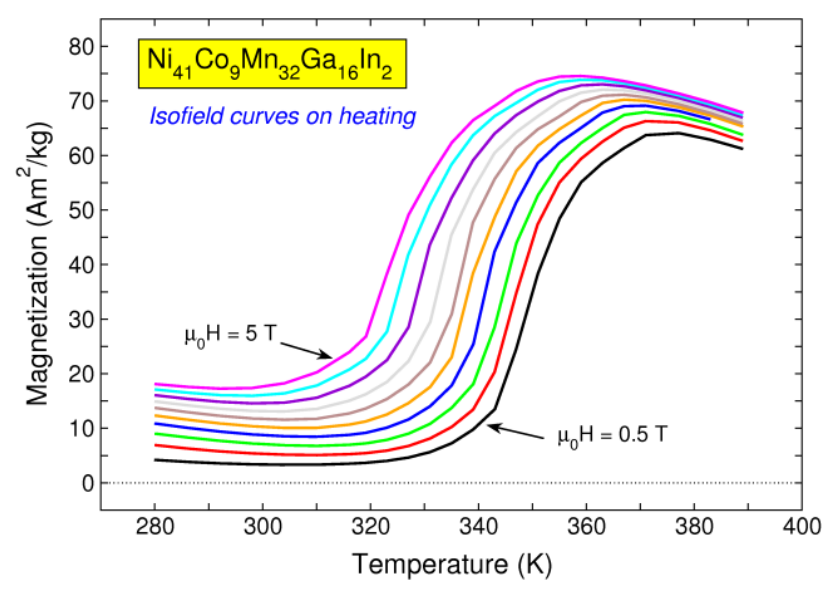

Figure 5. $M(T)$ isofield curves on heating around the martensitic (magnetostructural) transition with $0.5<\mu_{0} H<5 \mathrm{~T}$ showing the large $\Delta M$ in magnetization curves of Ni-Co-MnGa-In alloys. Data provided by F. Albertini [26, 27].

The resulting adiabatic temperature change $\Delta T_{a d}$ for this alloy is still quite small $(1.6 \mathrm{~K})$ in spite of the large change of $M$ showing that the magnetocaloric effect (MCE) needs further optimization compared to the classical Gd-Si-Ge, Fe-Rh and La-Fe-Si MCE materials, which all display $\Delta T_{a d}$ of about $13 \mathrm{~K}$ and a large isothermal entropy change $\Delta S_{i s o}$. There have been many attempts to improve the MCE in Heusler and other intermetallics which has been highlighted in the literature [31]. A discussion of the magnetostructural transition and adiabatic temperature variation in poly-crystal and single crystal Ni-Mn-Ga alloys can be found in [32].

It is very interesting to note that the binary system $\mathrm{Fe}$ $\mathrm{Rh}$ also displays a large jump of the magnetization which can be considered as a relict of the magnetostructural transition of the ternary $\mathrm{Rh}\left(\mathrm{Fe}_{1-\mathrm{x}} \mathrm{Pd}_{\mathrm{x}}\right)$ alloy system for vanishing $\mathrm{Pd}$ concentration [33]. See also discussion of $\mathrm{FePd}, \mathrm{MnRh}$ and $\mathrm{MnPd}$ compounds in comparison to FeRh [34]. The recent publications regarding the magnetization dynamics across the first order phase transition in FeRh thin films [35] and the temperature induced metamagnetic transition and domain structures of single-crystalline $\mathrm{FeRh}$ thin films on $\mathrm{MgO}(100)$ [36] show the ongoing interest in this classical magnetocaloric material [37]. Magnetostructural transition has also been discussed for B2-ordered FeRh epilayers which show coexistence of lattice expanded and contracted phases in spatially different regions [38] as well as coexistence of FM and AFM in the epilayers [39].

Figure 6 shows the existence of a magnetostructural transition in the Fe-Rh-Pd alloys. Regarding the magnetization behavior of FeRh in Fig. 7, we find a striking similarity to the variation of $M(T)$ over the magnetostructural transition in Heusler alloys (as in Fig. 5). We are currently performing calculations of the magnetocaloric effect in FeRh and its alloys. 


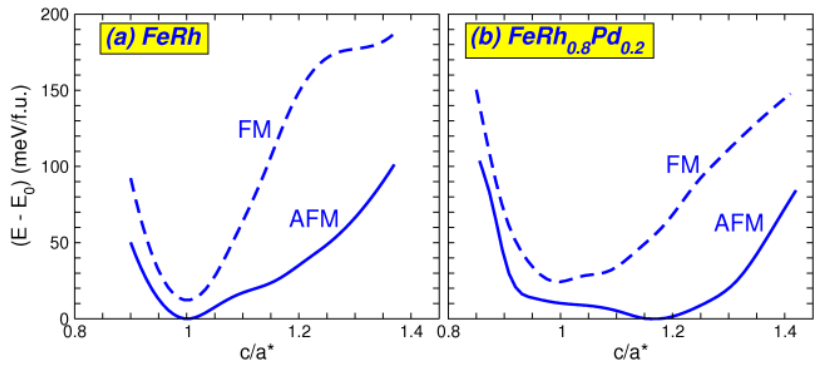

Figure 6. Calculated total energies of $\mathrm{Fe}\left(\mathrm{Rh}_{1-\mathrm{x}} \mathrm{Pd}_{\mathrm{x}}\right)$ for (a) $x=0$ and (b) $x=0.2$ for FM and AFM alignment (AF3 type stacking of (100) AFM layers in [001] direction, neighboring Fe layers couple AFM like) as a function of $c / a^{*}$, where $a^{*}$ is the lattice constant of $\mathrm{CuAu}-\mathrm{I}$ type ordered structure which gives the $\mathrm{CsCl}$ structure for $\mathrm{c} / \mathrm{a}^{*}=1$. (b) shows the structural transition for the AFM solution with the minimum of energy moving from $c / a^{*}=$ 1 to $c / a^{*}>1$. The virtual crystal approximation (VCA) has been used in the calculations with $a^{*}=3 \AA$ which is very close to the observed one, $a^{*}=2.99 \AA$ (data from [33]). For larger $x(x=$ $0.525)$ the FM phase with orthorhombic structure becomes the ground state for $\mathrm{Fe}\left(\mathrm{Rh}_{1-\mathrm{x}} \mathrm{Pd}_{\mathrm{x}}[37]\right.$.

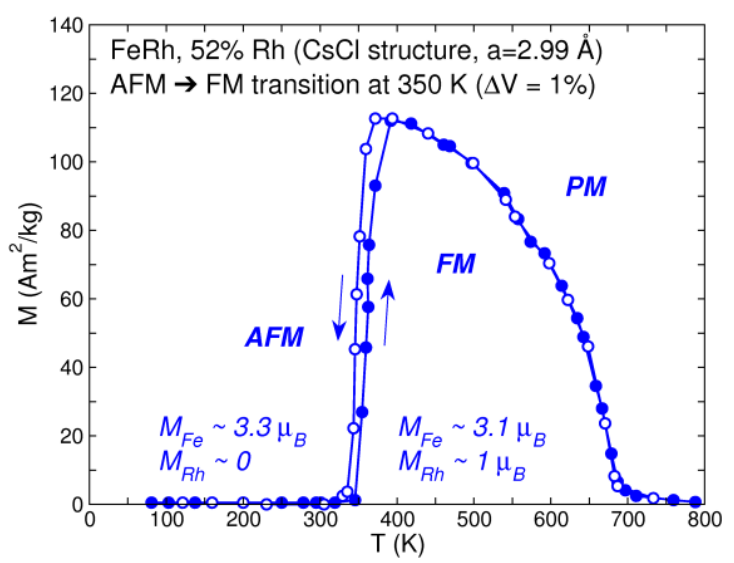

Figure 7. $M(T)$ curves of Fe-Rh on cooling and heating in zero magnetic field [19]. The magnetic collapse at the FM-AFM transition shows similarities with the corresponding large change of magnetization at the magnetostructural transition. Compare also the results of neutron scattering experiments [40]. The FM alignment is within the (111) planes and the AFM alignment is between (111) planes. Ab initio calculations essentially confirm this. The AFM type of order yielding the lowest energy state, is of AF3 type [33].

Note that similar magnetization curves as in Fig. 7 have also been obtained for the anti-perovskite $\mathrm{Mn}_{3} \mathrm{GaC}$ [41].

The large change of the magnetization, which is associated with the metamagnetic phase gives rise to a large inverse magnetocaloric effect compared with the conventional MCE at the Curie temperature.

\section{The atomistic origin of metamagnetic transitions in magnetic intermetallics}

In the same way as we find magnetism dominated by competing FM-AFM interactions in the nonstoichiometric Heusler $\mathrm{Ni}_{50} \mathrm{Mn}_{30} \mathrm{Ga}_{20}$ alloys as shown in Fig. 4(b), we observe coexistence of such interactions in
FeRh materials, too. Figure 8 shows corresponding results for the element-resolved magnetic coupling constants of FeRh. The corresponding orbital resolved $J_{i j}$ have also been calculated recently.

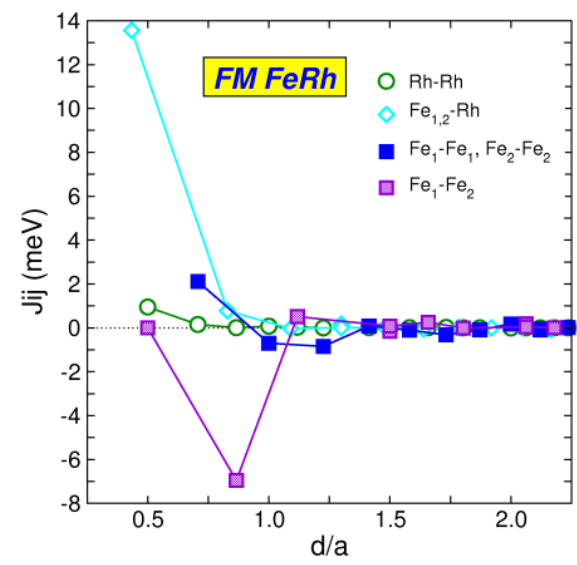

Figure 8. $A b$ initio results for the magnetic coupling constants calculated for the ferromagnetic reference state $(a=6.0 \AA$ is the lattice constant of the AFM structure). $\mathrm{Fe}_{2}$ is the next nearest $\mathrm{Fe}$ which flips its spin when the system undergoes the FM-AFM transition.

In conclusion, there is similarity between Fe-Rh and Ni-Mn-(Ga, In) alloys regarding the coexistence of FM and AFM interactions. These arise from the competing exchange interactions involving the $e_{g}$ and $t_{2 g}$ orbitals. With respect to the AFM-FM transition at the magnetostructural transition in Ni-Mn-In, we may interpret this (in much the same way as in Invar alloys) as a low-spin-to-high-spin (LS-HS) transition. Indeed, the LS-HS scenario shows up when we calculate the energy (with energetically nearly degenerate LS and HS states) or the individual magnetic moments of the atoms as a function of fixed magnetization per unit cell. This so called fixed spin moment method has been successfully used to describe magnetic anomalies in magnetic transition metal compounds. Figure 9 shows, e.g., the existence of a low-spin-high-spin (LS-HS) transition in $\mathrm{Ni}_{50} \mathrm{Mn}_{37.5} \mathrm{In}_{12.5}$ (using a cubic 16-atom supercell of $\mathrm{Ni}_{8} \mathrm{Mn}_{6} \mathrm{In}_{2}$ ).

A similar LS-HS scenario as in Fig. 9 is achieved for other disordered magnetic Heusler alloys, too. Also, if in addition to the fixed spin moment condition, we constrain other moments to undergo a spin-flip transition, we observe many energetically close lying LS states as shown in Fig. 10 for the case of $\mathrm{Ni}_{7} \mathrm{Co}_{1} \mathrm{Mn}_{7} \mathrm{Sn}_{1}$.

In the following we show results for the magnetocaloric effect calculated with the help of $a b$ initio calculations and Monte Carlo simulations [22, 29] in comparison to experimental data. In the Monte Carlo study we use an effective spin model which includes elastic and mangetoelastic interactions. We limit the discussion to the case of Co-doped Ni-(Co)-Mn-In, for which it is known that Co enhances considerably the MCE. Regarding the spin-spin interactions, we have used a Potts model, which allows to keep track of the various spin multiplicities such as $\mathrm{S}=5 / 2$ for $\mathrm{Mn}^{3+}, \mathrm{S}=1$ for $\mathrm{Ni}^{2+}, \mathrm{S}=3 / 2$ for $\mathrm{Co}^{2+}$ and $\mathrm{S}=4 / 2$ for $\mathrm{Cr}^{2+}$. For details, see $[22,24,29]$. 


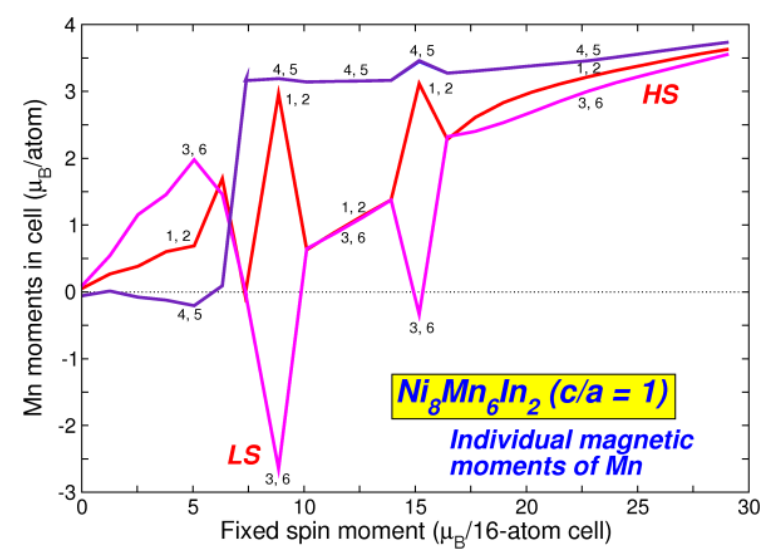

Figure 9. Variation of magnetic momens of the $6 \mathrm{Mn}$ atoms in the 16-atom supercell of austenitic $\mathrm{Ni}_{8} \mathrm{Mn}_{6} \mathrm{In}_{2}$ as a function of the fixed magnetization of the cell. The two $\mathrm{Mn}$ on the In sublattice, labeled Mn3 and Mn6, have their spins flipped leading to the low-spin state (LS), which is nearly energetically degenerate with the high-spin state (HS), see Fig. 10 .

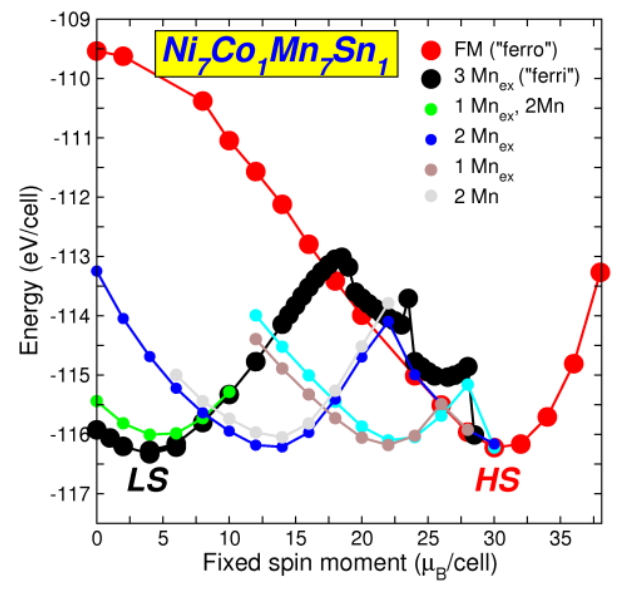

Figure 10. Energy variation of LS and HS states for the 16atom unit cell of austenitic $\mathrm{Ni}_{7} \mathrm{Co}_{1} \mathrm{Mn}_{7} \mathrm{Sn}_{1}$ as a function of the fixed magnetization of the cell. The three $\mathrm{Mn}$ on the $\mathrm{Sn}$ sublattice have their spins flipped leading to the low-spin state (LS: "ferri"), which is nearly energetically degenerate with the high-spin state (HS: "ferro"). Shown are other LS states achieved by different spin reversals, for example, $1 \mathrm{Mn}_{\mathrm{ex}}, 2 \mathrm{Mn}$, which means $1 \mathrm{Mn}$ on the Sn sublattice and $2 \mathrm{Mn}$ on their regular sublattice have their spins reversed. Note, however, that this has been obtained without relaxation of atomic positions.

\section{Magnetocaloric effect of metamagnetic $\mathrm{Ni}_{45} \mathrm{Co}_{5} \mathrm{Mn}_{37} / \mathrm{n}_{13}$ alloys}

The structural and magnetic properties (including magnetic exchange coupling constants) have been calculated using $a b$ initio method whereas the thermodynamic properties and MCE have been obtained from Monte Carlo simulation, using

$$
\Delta T_{a d}(T, H) \approx-T \frac{\Delta S_{\operatorname{mag}}(T, H)}{C(T, H)}
$$

whereby the entropy change across the magnetostructural transition in an external magnetic field is obtained by integrating the specific heat (using a model Hamiltonian which consists of magnetic, elastic and magnetoelastic terms, see [42]). In Eq. (1), the specific heat is the total specific heat (magnetic, electronic plus lattice part).

Figure 11 shows the final result for the conventional and inverse entropy and adiabatic temperature changes for $\mathrm{Ni}_{45} \mathrm{Co}_{5} \mathrm{Mn}_{37} \mathrm{In}_{13}$. The result compares well with the experimental data of Liu et al. [43]. In summary, we may say that the large jump of the magnetization at the first order magnetostructural transformation leads to the large entropy and adiabatic temperature changes. Cobalt enhances the magnetization of the austenitic phase favoring the large change of magnetization at the martensitic transformation and simultaneously leads to the undercooling effect of the austenitic phase.

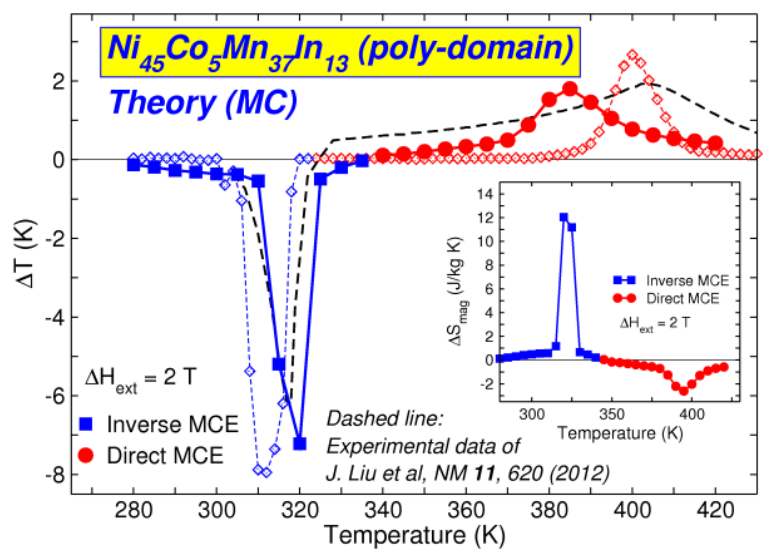

Figure 11. Adiabatic temperature change of the direct (filled circles) and inverse (filled squares) magnetocaloric effect of polycrystalline $\mathrm{Ni}_{45} \mathrm{Co}_{5} \mathrm{Mn}_{37} \mathrm{In}_{13}$ as obtained from the extended spin model. Diamonds mark results for a single domain phase while the inset shows the variation of the entropy [29].

The magnetic cooling effect shown here does not yet reach the values of the $\mathrm{Gd}, \mathrm{La}$ or Fe-Rh compounds but values tend already to reach $10 \mathrm{~K}$. If, in addition, the problem of magnetic and thermal hysteresis can be solved, these Heusler alloys appear to be attractive materials for future refrigeration technologies.

\section{Conclusion}

The combined effort of using $a b$ initio tools in combination with Monte Carlo methods allows to establish phase diagrams as well as functional materials properties at finite temperatures such as the magnetocaloric effect of intermetallics. The results show agreement with experiment and also allow predictions of how to improve the functionalities of these 'intelligent' materials.

The authors P.E., A.G. M.A. and A.Ç. acknowledge support by the DFG through the Priority Programme 1599 on "Ferroic Cooling", and M.E.G. by SFB TRR80. 


\section{References}

1. K. Otsuka, M. Wayman, Shape Memory Materials (Cambridge University Press, Cambridge, UK, (1998); K. Otsuka, X. Ren, Prog. Mater. Sci. 50, 5011 (2005).

2. S. Sarkar, X. Ren, K. Otsuka, Phys. Rev. Lett. 95, 205702 (2005) ; Y. Wang, X. Ren, K. Otsuka, Phys. Rev. Lett. 97, 225703 (2006); J. Lu, G. T. Martinez, S. Van Aert, D. Schryvers, Phys. Status Solidi B 251, 2034 (2014).

3. M. Acet, L. Mañosa, A. Planes, in Handbook of Magnetic Materials (2011), vol. 19, pp. 231-289.

4. K. Ullako, J. K. Huang, C. Kantner, R. C. O’Handley, V. V. Kokorin, Appl. Phys. Lett. 69, 1966 (1996).

5. T. Krenke, E. Duman, M. Acet, E. F. Wassermann, X. Moya, L. Mañosa, A. Planes, E. Suard, B. Ouladdiaf, Phys. Rev. B 75, 104414 (2007).

6. R. Kainuma, Y. Imano, W. Ito, Y. Sutou, H. Morito, S. Okamoto, O. Kitakami, K. Oikawa, A. Fujita, T. Kanomata, K. Ishida, Nature 439, 957 (2006).

7. F. X. Hu, G. Shen, J. R. Sun, Appl. Phys. Lett. 76, 3460 (2000).

8. T. Krenke, E. Duman, M. Acet, E. F. Wasermann, X. Moya, L. Mañosa, A. Planes, Nature Mater. 4, 450 (2005).

9. L. Pareti, M. Solzi, F. Albertini, A. Paoluzi, Euro. Phys. J. B 32, 303 (2003).

10. L. Mañosa, D. González-Alonso, A. Planes, E. Bonnot, M. Barrio, J.-L. Tamarit, S. Aksoy, M. Acet, Nature Mat. 9, 478 (2010).

11. P. O. Castillo-Villa, D. E. Soto-Parra, J. A. MatutesAquino, R. A. Ochoa-Gamboa, A. Planes, L. Manosa, D. Gonzáles-Alonso,M. Stipcich, R. Romero, D. Rios-Jara, H. Flores-Zuniga, Phys. Rev. B 83, 174109 (2011).

12. M. S. Lund, J. W. Dong, J. Lu, Y. Dong, C. J. Palmstrom, C. Leighton, Appl. Phys. Lett. 80, 4798 (2002).

13. Z. Li, C. Jing, J. Chen, S. Yuan, S. Cao, Z. Zhang, Appl. Phys. Lett. 91, 112505 (2007).

14. V. K. Sharma, M. K. Chattopadhyay, S. B. Roy, Phys. Rev. B 76, 140401R (2007).

15. D. Y. Cong, S. Roth, J. Liu, Q. Luo, M. Potschke, C. Hurrich, L. Schultz, Appl. Phys. Lett. 96, 112504 (2010).

16. Y. Wang, C. Huang, H. Wu, J. Gao, S. Yang, D. Wang, X. Ding, X. Song, X. Ren, Appl. Phys. Lett. 102, 141909 (2013).

17. V. K. Pecharsky, K. A. Gschneidner, Jr., Phys. Rev. Lett. 78, 4494 (1997).

18. F. X. Hu, B. G. Shen, J. R. Sun, Z.H. Cheng, G. H. Rao, X. X. Zhang, Appl. Phys. Lett. 78, 3675 (2001).

19. J. S. Kouvel and C. C. Hartelius, J. Appl. Phys, Suppl. 33, 1343 (1962).

20. P. Entel, M. E. Gruner, D. Comtesse, M. Wuttig, JOM 65, 1540 (2013).

21. P. Entel, M. Siewert, M. E. Gruner, H. C. Herper, D. Comtesse, R. Arróyave, N. Singh, A. Talapatra, V. V. Sokolovskiy, V. D. Buchelnikov, F. Albertini, L.
Righi, V. A. Chernenko, Eur. Phys. J. B 86, 65 (2013).

22. V. V. Sokolovskiy, V. D, Buchelnikov, V. V. Khovaylo, S. V. Taskaev, P. Entel, J. Refrig. 37, 273 (2014).

23. A. Çakır, L. Righi, F. Albertini, M. Acet, M. Farle, S. Aktürk, J. Appl. Phys. 114, 183912 (2013).

24. P. Entel, M. E. Gruner, D. Comtesse, V. V. Sokolovskiy, V. D. Buchelnikov, Phys. Stat. Solidi B 251, 2135 (2014).

25. M. E. Gruner, S. Fähler, P. Entel, Phys. Stat. Solidi 251, 2067 (2014).

25. K. P. Bhatti, S. El-Khatib, V. Srivastava, R. D. James, C. Leighton, Phys. Rev. B 85, 134450 (2012).

26. G. Porcari, S. Fabbrici, C. Pernechele, F. Albertini, M. Buzzi, A. Paoluzi, J. Kamarad, Z. Arnold, M. Solzi, Phys. Rev. B 85, 024414 (2012).

27. S. Fabbrici, G. Porcari, F. Cugini, M. Solzi, J. Kamarad, Z. Arnold, R. Cabassi, F. Albertini, Entropy 16, 2204 (2014).

28. R. Kainuma, Y. Imano, W. Ito, H. Morito, Y. Sutou, K. Oikawa, A. Fujita, K. Ishida, S. Okamoto, O. Kitakami, T. Kanomata, Appl. Phys. Lett. 88, 192513 (2006).

29. D. Comtesse, M. E. Gruner, M. Ogura, V. V. Sokolovskiy, V. D. Buchelnikov, A. Grünebohm, R. Arróyave, N. Singh, T. Gottschall, O. Gutfleisch, V. A. Chernenko, F. Albertini, S. Fähler, P. Entel, Phys. Rev. B 89, 184403 (2014).

30. D. Comtesse, Thesis, University of Duisburg-Essen (2014).

31. K. G. Sandeman, Scripta Mater. 67, 566 (2012).

32. C. P. Sasso, M. Pasquale, L. Giudici S. Besseghini, E. Villa, T. A. Lograsso, D. L. Schlagel, J. Appl. Phys. 99, 08K905 (2006).

33. K. Uebayashi, H. Shimizu, H. Yamda, Mater. Trans. 47, 456 (2006).

34. H. Yamada, H. Shimizu, K. Yamamoto, K. Uebayashi, J. Alloy Comp. 415, 31 (2006).

35. F. Pressaco, Thesis, University of Regensburg (2013).

36. X. Zhou, Thesis, University of Köln (2013).

37. S. Yuasa, H. Miyajima, Y. Otani, G. Masada, N. Wakabayahi, K. Tajima, J. Phys. Soc. Jpn. 64, 3153 (1995).

38. M. A. de Vries, M. Loving, M. McLaren, R. M. D. Brydson, X. Liu, S. Langridge, L. H. Lewis, C. H. Marrows, Appl. Phys. Lett. 104, 232407 (2014).

39. R. Fan, C. J. Kinane, T. R. Charlton, R. Dorner, M. Ali, M. A. de Vries, R. M. D. Brydson, C. M. Marrows, B. J. Hickey, D. A. Arena, B. K. Tanner, G. Nisbet, S. Langridge, Phys. Rev. B 82, 184418 (2010).

40. G. Shirane, R. Nathans, C. W. Chen, Phys. Rev. 134, A1547 (1964).

41. Ö. Çakır, M. Acet, Appl. Phys. Lett. 100, 202404 (2012).

42. V. V. Sokolovskiy, O. Pavlukhina, V. D. Buchelnikov, P. Entel, J. Phys. D : Appl. Phys. 47, 425002 (2014).

43. J. Liu, T. Gottschall, K. P. Skokov, J. D. Moore, O. Gutfleisch, Nature Mater. 11, 620 (2012). 